\title{
Quantitative differences in the proteomic composition of the blood serum of patients with simple and paranoid schizophrenia
}

\author{
Smirnova Liudmila \\ Laboratory of Molecular Genetics and \\ Biochemistry \\ Mental Health Research Institute \\ of Tomsk National Research Medical \\ Center \\ Tomsk, Russia \\ lpsmirnova@yandex.ru \\ Seregin Alexandr \\ Laboratory of Molecular Genetics and \\ Biochemistry \\ Mental Health Research Institute of \\ Tomsk National Research Medical \\ Center \\ Tomsk, Russia \\ lena-312tom@yandex.ru
}

\author{
Zgoda Victor \\ Laboratory of systems biology \\ Institute of Biomedical Chemistry \\ Moscow, Russia \\ vic@ibmh.msk.su \\ Semke Arkadiy \\ Department of Endogenous Disorders \\ Mental Health Research Institute of \\ Tomsk National Research Medical \\ Center \\ Tomsk, Russia \\ arkan1959@mail.ru
}

\author{
Dmitrieva Elena \\ Laboratory of Molecular Genetics and \\ Biochemistry \\ Mental Health Research Institute of \\ Tomsk National Research Medical \\ Center \\ Tomsk, Russia \\ lena-312tom@yandex.ru \\ Ivanova Svetlana \\ Laboratory of Molecular Genetics and \\ Biochemistry \\ Mental Health Research Institute of \\ Tomsk National Research Medical \\ Center \\ Tomsk, Russia \\ ivanovaniipz@gmail.com
}

\begin{abstract}
In the present study we analyzed the protein spectrum of blood in schizophrenic patients with simple and paranoid form of diagnosis. A set of proteins in schizophrenia were typically associated with processes, which are responsible for protein synthesis and the processes of transduction and translation; immune response, oxidative stress, apoptosis and cell communication. Significant increase in the number of RIPK1, mGluR6 receptors in blood serum between patients and healthy controls was revealed by comparative analysis. Besides, the amount of mGluR6 was significantly higher in patients with simple form of schizophrenia compared with paranoid schizophrenia patients $(p=0.021)$. A quantitative assessment of the specific minor proteins that we studied, using labeled standard peptides, demonstrated an increase in their number depending on the severity of the disease (a simple form of schizophrenia with leading negative symptoms).
\end{abstract}

Keywords - biomarker, schizophrenia, proteome, mass spectrometry, serum

\section{Introduction}

The study of mental disorders becomes more relevant in the 21 st century. Schizophrenia refers to socially significant diseases, since it is a chorionic disease that begins at a young age. Its etiology and pathogenesis, response to treatment and its consequences are not yet completely understood. As a rule, only anamnestic and clinical psychopathological data are used for diagnostics. Patients, entering a psychiatric clinic for the first time, have particular difficulties in making a diagnosis. At present, the search for peripheral biomarkers, which can be used for differential diagnosis and prognosis of therapy (theranostic), becomes particularly important. Work on proteomic analysis in schizophrenic patients was carried out mainly on post-mortem material. However, so far, the search for markers of mental disorders has not been successful. The identification of peripheral biomarkers is an important step towards personalized medicine [1][2].

\section{Methods}

The protein spectrum of the blood serum was analyzed in following groups: 20 patients ( 9 men, 11 women, aged 32 [26, $41]$ years and a disease duration of $7[4,16]$ years) with acute paranoid schizophrenia (F20.0); 15 patients (6 men, 9 women, aged $34[29,44]$ years and a disease duration of $10[8,20]$ years) with simple schizophrenia (F20.6) and 10 healthy people, the average age of 32,6 [22; 37] years. The patients were hospitalized in the Department of Endogenous Disorders of the Research Institute of Mental Health (MHRI) of the Tomsk National Research Medical Centre (NRMC, Tomsk). Blood serum samples were obtained from most patients in acute state before the start of the study. This was done in order to get clean samples before starting their course of therapy. The serum was purified by affinity chromatography from six major proteins (albumin, immunoglobulin G, immunoglobulin A, antitrypsin, transferrin and haptoglobin). In the next step the purified proteins were concentrated to 1 ml using Amicon Ultra-0.5 ultrafilters (MILLIPORE) at 3 $\mathrm{kDa}$ and separated by vertical electrophoresis in a $12 \%$ polyacrylamide gel according to the Lemmli method. Then, after trypsinolysis and peptide extraction from the gel, the proteins were identified by HPLC / mass spectrometry on Qexactive HF mass spectrometer (Thermo Scientific). Mass spectrometry was performed in Centre of Collective Usage "Human proteome» of IBMC, Moscow.

The mass spectrometric data were analyzed with the MaxQuant software (version 1.6.3.4). The Andromeda search engine was used to search the MS/MS spectra against the UniProt human database combined with 262 common contaminants and concatenated with the reversed versions of all sequences. Only proteins quantified with at least two peptides were considered for quantitation. Quantitative LCMS-SRM analysis was performed on QQQ TSQ Vantage (Thermo Scientific) equipped with a nano-electrospray ion source. Each sample was analyzed for five times using Dionex UltiMate 3000 RSLCnano System Series (Thermo Scientific). 
Statistical analysis was performed using STATISTICA version 10.0 (StatSoft, Tulsa, OK, USA). The KolmogorovSmirnov test was used to determine whether the data were normally distributed. Non-parametric Mann-Whitney U-test and Fisher's exact test with Yates' correction was used to check the statistical significance between-group differences. Kruskal-Wallis one-way analysis of variance (ANOVA) and median test were used for comparative data analysis between three groups. Differences and correlations were considered significant at p-value.

\section{Results}

In the work, a qualitative and quantitative analysis of the proteomes of the blood serum between patients with two different forms of schizophrenia (simple and paranoid) and healthy individuals was carried out. In patients with a paranoid form of schizophrenia, 5202 proteins were identified. Among them 1097 proteins showed significant differences with the proteins of healthy individuals. Patients with a simple form of schizophrenia identified 4098 proteins. Among them 574 proteins showed significant differences with control. Statistically significant differences in 302 proteins were obtained between patients with simple and paranoid forms of schizophrenia. Most of them from set of proteins in schizophrenia were typically associated with processes, which are responsible for protein synthesis and the processes of transduction and translation; immune response, oxidative stress, apoptosis and cell communication.

For further research by the method of quantitative mass spectrometry using labeled peptide standards, several proteins involved in neurogenesis and proteins of neuronal receptors were selected: NMDAZ1, RIPK1, mGluR6, DCLK1. Kruskal-Wallis test ANOVA by Ranks showed significant differences between three studied groups for protein DCLK1 $(p=, 0118)$ and, accordingly, significant pairwise differences between all the studied groups. In the blood serum of patients in the total group of schizophrenia, a significant increase in the number of RIPK1, mGluR6 receptors was revealed in comparison with healthy people. In addition, the amount of mGluR6 was significantly higher in patients with a simple form of schizophrenia compared with paranoid form patients $(p=0.021)$. In the total group of patients with schizophrenia, divided accordingly by the prevailing symptoms, a more than twofold increase in the number of RIPK1 was revealed in patients with leading negative symptoms. Thus, most of the specific proteins that we studied that has been identified in patients with schizophrenia using screening were significantly increased depending on the clinical symptoms.

\section{Conclusions}

Saia-Cereda with co-workers describes a large amount of proteins typical for brain proteome in schizophrenia, as well as for major depressive disorder (MDD) [3]. Through comparative analysis of proteome profiles between schizophrenia, $\mathrm{BD}$, and $\mathrm{MDD}$, only 30 proteins were similarly changed in all three disorders. Hence, small overlapping between changes in protein levels typical for major mental disorders can be a feature maintaining specificity of every disease at proteome level.

A quantitative assessment of the specific minor proteins that we studied using labeled standard peptides showed an increase in their number depending on the severity of the disease (a simple form of schizophrenia, leading negative symptoms). Thus, the identified proteins have pathogenetic and prognostic significance.

Hence, one may suppose that finally the efforts must not be concentrated on the search for a specific protein, but rather a protein set (panel) must be revealed reflecting the main pathogenetic mechanisms and serving as a starting point for diagnosis and prognosis of mental disorder development [4].

\section{ACKNOWLEDGMENT}

This work was supported by the RSF grant No.18-1500053 "Search for peripheral markers associated with impaired myelination of the brain and the pathogenesis of the disease in schizophrenia" 2018-2020.

\section{REFERENCES}

[1] Semke A.V., Fedorenko O.Yu., Lobacheva O.A., Rakhmazova L.D., Kornetova E.G., Smirnova L.P., Mikilev F.F., Schigoreva Yu.G. Clinical, epidemiological and biological background of the adaptation of patients with schizophrenia as the basis of a personalized approach to antipsychotic therapy (2015) Siberian Bulletin of Psychiatry and Addiction. 3(88): 19-25.

[2] Loginova L.V., Smirnova L.P., Koval V.V., Fedorova O.S., Semke A.V., Ivanova S.A. Mass-spectrometric analysis of proteins of blood serum of patients with schizophrenia (2011) Bulletin of the Siberian Branch of the Russian Academy of Medical Sciences. 31(6): 63-68.

[3] Saia-Cereda V.M., Cassoli J.S., Martins-de-Souza D., Nascimento J.M. (2017) Psychiatric disorders biochemical pathways unraveled by human brain proteomics. Eur Arch Psychiatry Clin Neurosci. 26: 3-17.

[4] Loginova L.V., Smirnova L.P., Seregin A.A., Dmitrieva E.M., Mazin E.V., Simutkin G.G. To the question of the search for biomarkers in bipolar affective disorder (2014) Bulletin of the Ural Medical Academic Science. 3(49): 139-141. 